\title{
Intervenções de enfermagem para o cuidado de pacientes com artrite: revisão integrativa da literatura
}

\author{
Nursing interventions for the care of patients with arthritis: an integrative review \\ Intervenciones de enfermería para el cuidado de pacientes con artritis: revisión integradora
}

\section{Daniella Soares dos Santos', Emília Campos de Carvalho"}

' Universidade de São Paulo, Escola de Enfermagem de Ribeirão Preto, Programa Interunidades de Doutoramento em Enfermagem (Doutoranda). Ribeirão Preto-SP. Universidade de Brasília, Faculdade de Ciências da Saúde, Departamento de Enfermagem. Brasília-DF, Brasil.

"Universidade de São Paulo, Escola de Enfermagem de Ribeirão Preto, Departamento de Enfermagem Geral e Especializada, Programa Interunidades de Doutoramento em Enfermagem. Ribeirão Preto-SP, Brasil.

Submissão: 03-06-2011 Aprovação: 20-11-2012

\section{RESUMO}

Esta revisão integrativa buscou analisar a produção científica relacionada aos cuidados de enfermagem para pacientes com artrite. Foram incluídos 12 estudos experimentais, randomizados e controlados, publicados nas bases de dados CINAHL, MEDLINE, SciELO e LILACS, utilizando os descritores controlados "arthritis" e "nursing". Os resultados apontaram a efetividade de musicoterapia, estimulação elétrica neuromuscular, toque terapêutico e imagem guiada associada a relaxamento, para o tratamento da dor. Ser atendido por enfermeira especialista aumentou a satisfação com o atendimento, melhorou o impacto da doença e aumento a procura por serviços de saúde. Programas educativos específicos para portadores de artrite estimularam a prática de exercícios físicos e aqueles direcionados a pessoas com problemas crônicos em geral mostraram-se efetivos para controlar a dor e a incapacidade funcional. Concluímos que existe um conjunto de intervenções que podem subsidiar a prática de enfermagem baseada em evidências junto aos idosos com artrite.

Descritores: Artrite; Cuidados de Enfermagem; Idoso.

\section{ABSTRACT}

This integrative review aimed to analyze the scientific literature related to nursing care for patients with arthritis. Twelve experimental studies were included, randomized and controlled, published in CINAHL, MEDLINE, SciELO and LILACS databases, using the controlled keywords "arthritis" and "nursing". The results indicated the effectiveness of music therapy, neuromuscular electrical stimulation, therapeutic touch and guided imagery associated with relaxation, for the treatment of pain. Being treated by a specialist nurse increased satisfaction with care, improved the impact of the disease and increased the demand for health services. Specific educational programs for people with arthritis stimulated the practice of physical exercises and those targeted to people with chronic conditions in general were effective to control pain and functional disability. We conclude that there is a set of interventions that can support the practice of evidence-based nursing for the elderly people with arthritis.

Key words: Arthritis; Nursing Care; Aged.

\section{RESUMEN}

Esta revisión integradora tuvo como objetivo analizar la literatura científica relacionada con los cuidados de enfermería en pacientes con artritis. Se incluyeron 12 estudios experimentales, los ensayos controlados aleatorios, publicados en las bases de datos CINAHL, MEDLINE, LILACS y SciELO, utilizando las palabras clave controladas "artritis" y "enfermería". Los resultados indican la efectividad de la musicoterapia, la estimulación eléctrica neuromuscular, toque terapéutico y la imaginación guiada asociada con la relajación, para el tratamiento del dolor. Ser tratado por una enfermera especialista aumentó la satisfacción con la atención, la mejora del impacto de la enfermedad y aumentar la demanda de servicios de salud. Los programas específicos de educación para personas con artritis estimulado ejercicios físicos y los destinados a las personas con enfermedades crónicas en general son eficaces para controlar el dolor y la discapacidad funcional. Llegamos a la conclusión de que existe un conjunto de intervenciones que pueden apoyar la práctica de la enfermería basada en la evidencia para los ancianos con artritis.

Palabras clave: Artritis; Atención de Enfermería; Anciano.

Trabalho desenvolvido no Grupo de Pesquisa Enfermagem e Comunicação da EERP - USP/ Centro Colaborador da OMS para o desenvolvimento da pesquisa em Enfermagem.

\section{AUTOR CORRESPONDENTEＤaniella Soares Santos E-mail: danienf@usp.br}




\section{INTRODUÇÃO}

A dor figura como uma das principais causas para o abandono das atividades da vida diária e pela procura por serviços de saúde, sobretudo na população idosa ${ }^{(1)}$, levando à perda da capacidade funcional e da independência, além de outras formas de comprometimento como depressão e isolamento social ${ }^{(2)}$.

Entre as principais causas de dor na população idosa estão as doenças debilitantes como a artrite ${ }^{(3)}$, doença inflamatória degenerativa das articulações que se instala insidiosa e progressivamente com o passar dos anos( ${ }^{(4)}$.

De maneira geral a artrite se inicia com perda da superfície da cartilagem hialina podendo apresentar-se acompanhada de quadro inflamatório (artrite reumatoide) e comprometimento da extremidade óssea (osteoartrite), ocorrendo principalmente nas articulações mais expostas à sobrecarga de peso do corpo. O tratamento requer procedimentos cirúrgicos e órteses ${ }^{(5)}$, atendimento multiprofissional por tempo prolongado $^{(6)}$, além do uso de medicamentos.

Nesse sentido, é necessário identificar os cuidados de enfermagem mais eficazes com base nas melhores em evidências disponíveis.

Em face desta problemática, realizamos uma revisão integrativa recortando, como objeto de estudo, a análise da produção científica sobre intervenções utilizadas no cuidado de pacientes com artrite, publicadas até Fevereiro de 2011. O objetivo proposto foi analisar a produção científica em relação às evidências dos resultados produzidos por cuidados de enfermagem direcionados a pessoas com artrite.

\section{MÉTODO}

Trata-se de uma revisão integrativa da literatura, recurso escolhido por permitir a obtenção de dados sobre um tema de interesse a partir da utilização de estudos realizados com rigor metodológico que sustentem tanto a prática quanto a pesquisa, baseada em evidências sólidas ${ }^{(7)}$.

Para a condução metodológica foram seguidas seis etapas conforme proposto na literatura ${ }^{(8)}$. Uma vez identificado o tema de interesse a seguinte questão norteadora foi elaborada: Quais são os cuidados de enfermagem que produzem resultados significativos/positivos na saúde das pessoas com artrite?

A busca foi realizada nas seguintes bases de dados: Cumulative Index to Nursing and Allied Health Literature (CINAHL), Scientific Electronic Library Online (SciELO), Literatura Internacional em Ciências da Saúde (MEDLINE) e Literatura Latino-Americana e do Caribe em Ciências da Saúde (LILACS), utilizando os descritores controlados "nursing" and "arthritis". Os critérios de inclusão utilizados na seleção dos artigos foram: estudos clínicos realizados com sujeitos idosos; delineamento experimental com randomização e controle; idiomas: inglês, espanhol ou português; publicados até 28/02/2011. Os estudos incluídos na amostra foram avaliados quanto ao rigor metodológico, por meio da aplicação de uma escala para verificação da qualidade metodológica, sendo atribuídos pontos que vão de 0 a 5 . Valores $=3$ são considerados de qualidade pobre ${ }^{(9)}$.

A Figura 1 apresenta o percurso metodológico seguido para seleção dos estudos incluídos na amostra.

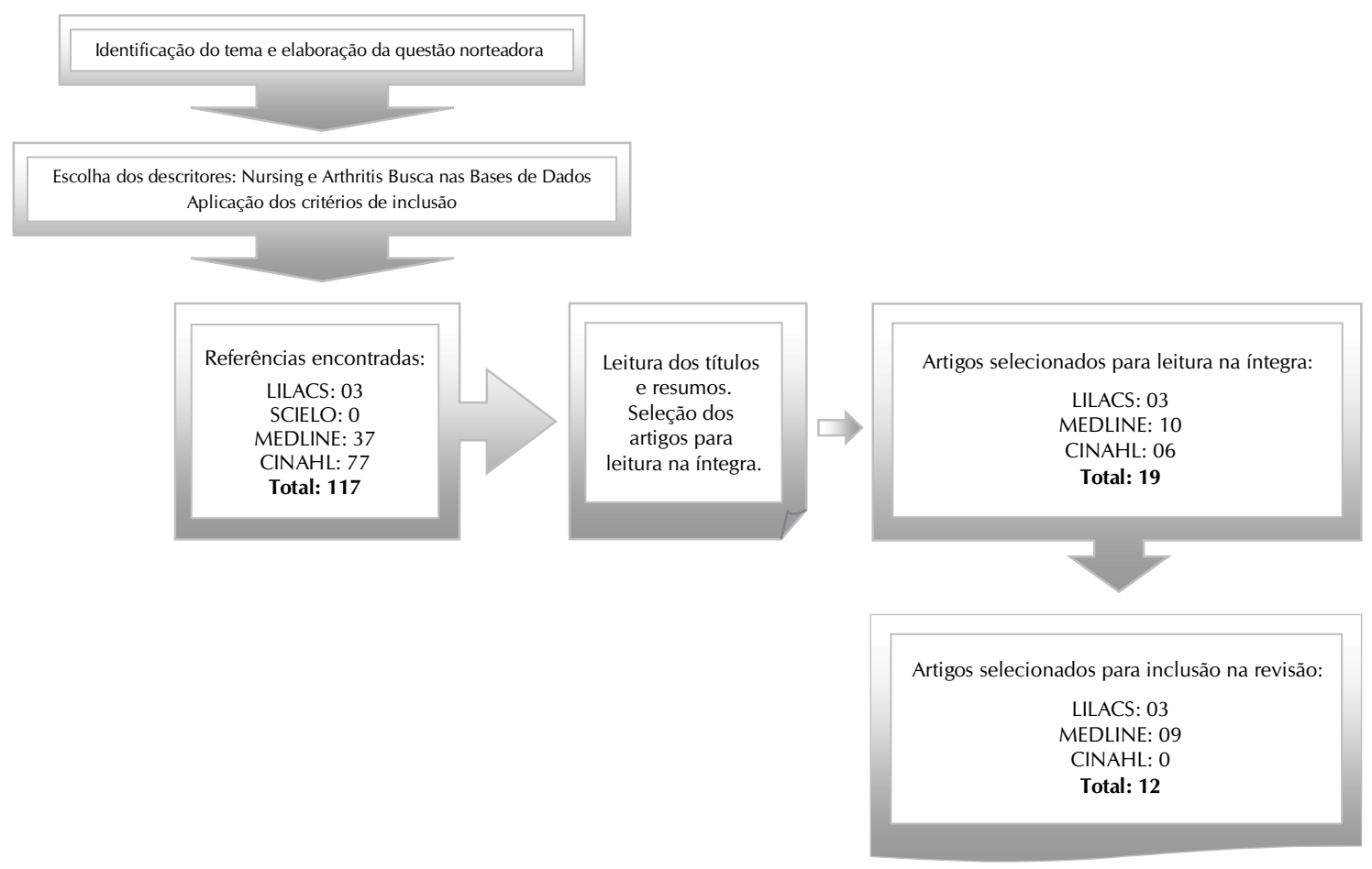

Figura 1 - Fluxograma da seleção amostral dos estudos incluídos na Revisão Integrativa. Ribeirão Preto, 2011. 


\section{RESULTADOS}

Dos 117 estudos identificados na busca às Bases de Dados, 12 foram selecionados para compor a amostra final (Figura 1). As demais publicações foram excluídas por não atenderem aos critérios de inclusão propostos neste estudo.

Quanto ao rigor metodológico, os estudos analisados obtiveram nota quatro nos quesitos randomização, cegamento, apresentação de perdas e exclusões na amostra, adequação do cegamento e da randomização.

A Tabela 1 apresenta os estudos incluídos na revisão segundo a autoria, objetivos, variáveis, método e principais resultados. O período de publicação dos estudos compreendeu de 1998 a 2010.

Tabela 1 - Distribuição dos estudos incluídos na revisão segundo primeiro autor, objetivos, variáveis, método e principais resultados.

\begin{tabular}{|c|c|c|c|c|}
\hline Autor & Objetivos & Variáveis & Método & Principais Resultados \\
\hline Gordon et al (10) & $\begin{array}{l}\text { Avaliar a } \\
\text { efetividade } \\
\text { do Toque } \\
\text { Terapêutico }\end{array}$ & $\begin{array}{l}\text { Bem estar, status } \\
\text { de saúde, dor. }\end{array}$ & $\begin{array}{l}\text { População: sujeitos internados em uma } \\
\text { instituição asilar. } \\
\text { Amostra: } 25 \text { sujeitos ( } 40-80 \text { anos). } \\
\text { Três grupos alocados de forma randomizada, } \\
\text { para receber TT, mímica (placebo) e cuidado } \\
\text { padrão, uma vez por semana, durante seis } \\
\text { semanas. }\end{array}$ & $\begin{array}{l}\text { Os sujeitos do grupo do TT } \\
\text { apresentaram diminuição da } \\
\text { intensidade da dor e melhora do } \\
\text { status funcional }\left(x^{2} ; p=0,001\right) \text {. } \\
\text { A avaliação do bem estar foi } \\
\text { qualitativa e considerou-se } \\
\text { melhora no grupo do TT. }\end{array}$ \\
\hline Gallez et al ${ }^{(11)}$ & $\begin{array}{l}\text { Avaliar a } \\
\text { efetividade de } \\
\text { uma terapia } \\
\text { complementar } \\
\text { espiritual. }\end{array}$ & $\begin{array}{l}\text { Dor, força } \\
\text { muscular, } \\
\text { flexibilidade } \\
\text { articular. }\end{array}$ & $\begin{array}{l}\text { População: Sujeitos portadores de artrite } \\
\text { atendidos em um ambulatório } \\
\text { de reumatologia. } \\
\text { Amostra: } 29 \text { sujeitos ( } 40 \text { - } 74 \text { anos). } \\
\text { Os sujeitos foram aleatoriamente alocados } \\
\text { em dois grupos para receber tratamento } \\
\text { médico convencional com ou sem } \\
\text { terapia espiritual por seis meses. }\end{array}$ & $\begin{array}{l}\text { Não foram encontradas } \\
\text { diferenças entre os grupos. }\end{array}$ \\
\hline Tijhuis et al ${ }^{(12)}$ & $\begin{array}{l}\text { Avaliar a } \\
\text { efetividade do } \\
\text { cuidado prestado } \\
\text { por enfermeira } \\
\text { especialista, } \\
\text { comparado com } \\
\text { equipe hospitalar } \\
\text { e de cuidado } \\
\text { diário. }\end{array}$ & $\begin{array}{l}\text { Status funcional } \\
\text { e de saúde, } \\
\text { qualidade de vida } \\
\text { e atividade da } \\
\text { doença. }\end{array}$ & $\begin{array}{l}\text { População: Sujeitos atendidos em } \\
\text { ambulatórios de reumatologia de } 6 \text { hospitais } \\
\text { acadêmicos e não acadêmicos } \\
\text { Amostra: } 210 \text { sujeitos ( } 28-85 \text { anos). } \\
\text { Três grupos alocados randomicamente para } \\
\text { receber cuidados: de enfermeira especialista, } \\
\text { de equipe de unidade de interação e de } \\
\text { equipe de atendimento ambulatorial. }\end{array}$ & $\begin{array}{l}\text { Os três grupos apresentaram } \\
\text { melhora no status funcional e } \\
\text { de saúde, qualidade de vida } \\
\text { e atividade da doença }\left(x^{2} ;\right. \\
\mathrm{p}<0,05) \text {. O grupo atendido } \\
\text { por enfermeira especialista } \\
\text { apresentaram maios satisfação } \\
\text { com o atendimento }\left(x^{2} ;\right. \\
p<0,001) \text { comparado com os } \\
\text { outros grupos. }\end{array}$ \\
\hline $\begin{array}{l}\text { McCaffrey, } \\
\text { Freeman }{ }^{(13)}\end{array}$ & $\begin{array}{l}\text { Avaliar o efeito } \\
\text { da música na } \\
\text { redução da dor. }\end{array}$ & Percepção da dor. & $\begin{array}{l}\text { População: Voluntários idosos portadores } \\
\text { de artrite de uma comunidade. } \\
\text { Amostra: } 66 \text { idosos (idade média: } 76 \text { anos) } \\
\text { Os sujeitos foram randomizados em dois } \\
\text { grupos para ouvir } 20 \text { minutos de música, } \\
\text { ou permanecerem em silêncio, uma vez } \\
\text { ao dia por duas semanas. }\end{array}$ & $\begin{array}{l}\text { Redução na percepção da } \\
\text { dor pelos sujeitos do grupo } \\
\text { experimental nos dias } 1,7 \text { e } \\
14 \text { (t-test; } p=0,001 \text {.) segundo } \\
\text { o Pain Rating Index e Visual } \\
\text { Analogue Scale. }\end{array}$ \\
\hline Tijhuis et al (5) & $\begin{array}{l}\text { Avaliar a } \\
\text { efetividade do } \\
\text { cuidado prestado } \\
\text { por enfermeira } \\
\text { especialista em } \\
\text { dois anos de } \\
\text { seguimento. }\end{array}$ & $\begin{array}{l}\text { Tratamento } \\
\text { médico, uso dos } \\
\text { serviços de saúde, } \\
\text { uso de novos } \\
\text { equipamentos de } \\
\text { saúde, número de } \\
\text { hospitalizações, } \\
\text { visitas à } \\
\text { enfermeira } \\
\text { especialista. }\end{array}$ & $\begin{array}{l}\text { População: Sujeitos atendidos em } \\
\text { ambulatórios de reumatologia de } 6 \text { hospitais } \\
\text { acadêmicos e não acadêmicos } \\
\text { Amostra: } 210 \text { sujeitos ( } 28-85 \text { anos) } \\
\text { Os sujeitos que participaram de estudo } \\
\text { anterior (Tijuis, 2002) foram seguidos } \\
\text { por dois anos e avaliados nas semanas } \\
12,26,52,78 \text { e } 104 \text {. }\end{array}$ & $\begin{array}{l}\text { O grupo atendido por } \\
\text { enfermeira especialista } \\
\text { apresentou maior procura por } \\
\text { atendimento profissional }\left(x^{2} ;\right. \\
p<0,05) \text {. Não houve diferença } \\
\text { entre os grupos quanto às } \\
\text { demais variáveis. }\end{array}$ \\
\hline
\end{tabular}




\begin{tabular}{|c|c|c|c|c|}
\hline Autor & Objetivos & Variáveis & Método & Principais Resultados \\
\hline Gaines et al (14) & $\begin{array}{l}\text { Avaliar os efeitos } \\
\text { da estimulação } \\
\text { elétrica } \\
\text { neuromuscular na } \\
\text { redução da dor. }\end{array}$ & $\begin{array}{l}\text { Dor, evolução da } \\
\text { doença. }\end{array}$ & $\begin{array}{l}\text { População: Voluntários portadores de } \\
\text { osteoartrite de joelho, saudáveis à } \\
\text { entrada no estudo. } \\
\text { Amostra: } 38 \text { sujeitos (idade média: } 70 \text { anos) } \\
\text { Randomização para receber tratamento com } \\
\text { estimulação elétrica neuromuscular mais } \\
\text { um programa de educação sobre artrite } \\
\text { ou o programa educacional apenas. }\end{array}$ & $\begin{array}{l}\text { Redução da dor no grupo } \\
\text { que recebeu p programa } \\
\text { educacional mais estimulação } \\
\text { elétrica após } 15 \text { minutos de } \\
\text { tratamento (t-test; } p<0,001) \text {. } \\
\text { Não houve diferença entre os } \\
\text { grupos quanto à evolução da } \\
\text { doença durante o seguimento. }\end{array}$ \\
\hline Ryan et al (15) & $\begin{array}{l}\text { Avaliar o } \\
\text { impacto de da } \\
\text { assistência de } \\
\text { uma enfermeira } \\
\text { reumatologista } \\
\text { sobre o bem } \\
\text { estar de sujeitos } \\
\text { submetidos a } \\
\text { monitorização } \\
\text { no uso de } \\
\text { medicamentos. }\end{array}$ & $\begin{array}{l}\text { Atividade } \\
\text { da doença e } \\
\text { habilidade do } \\
\text { paciente em } \\
\text { controlar a } \\
\text { doença. }\end{array}$ & $\begin{array}{l}\text { População: Sujeitos portadores de } \\
\text { artrite atendidos em um ambulatório } \\
\text { de reumatologia. } \\
\text { Amostra: } 71 \text { sujeitos (média de idade: } \\
57 \text { anos). } \\
\text { Os sujeitos foram randomizados } \\
\text { para serem atendidos por enfermeira } \\
\text { especialista ou staff. }\end{array}$ & $\begin{array}{l}\text { O grupo experimental } \\
\text { apresentou melhora nos escores } \\
\text { de impacto da doença ( } U \text {-test; } \\
\mathrm{p}=0,03) \text { e atividade da doença } \\
(U \text {-test; } \mathrm{p}=0,048) .\end{array}$ \\
\hline $\begin{array}{l}\text { Goeppinger } \\
\text { et al (16) }\end{array}$ & $\begin{array}{l}\text { Avaliar a } \\
\text { efetividade de } \\
\text { dois programas } \\
\text { educacionais para } \\
\text { pacientes com } \\
\text { artrite. }\end{array}$ & $\begin{array}{l}\text { Uso do serviço } \\
\text { de saúde, saúde } \\
\text { em geral, prática } \\
\text { de exercícios, } \\
\text { controle da } \\
\text { doença e da dor, } \\
\text { incapacidade } \\
\text { funcional. }\end{array}$ & $\begin{array}{l}\text { População: Voluntários afro-americanos } \\
\text { portadores de artrite } \\
\text { Amostra: } 416 \text { sujeitos (idade média: } 65 \text { anos). } \\
\text { Os sujeitos participaram de } 48 \text { workshops } \\
\text { que foram randomizados para tratarem } \\
\text { especificamente de artrite ou doenças } \\
\text { crônicas em geral. }\end{array}$ & $\begin{array}{l}\text { O programa específico para } \\
\text { manejo de artrite melhorou o } \\
\text { controle da doença, prática de } \\
\text { exercícios, e a saúde em geral } \\
\text { (t-test; } p<0,05) \text {. O programa } \\
\text { para doentes crônicos em geral } \\
\text { melhorou o controle da dor e a } \\
\text { incapacidade funcional (t-test; } \\
\text { p }<0,051) \text {. Ambos os grupos } \\
\text { apresentaram melhora na } \\
\text { saúde geral. }\end{array}$ \\
\hline $\begin{array}{l}\text { McDonald et } \\
\text { al (17) }\end{array}$ & $\begin{array}{l}\text { Avaliar se a } \\
\text { aplicação de } \\
\text { um questionário } \\
\text { com perguntas } \\
\text { abertas e fechadas } \\
\text { antes da consulta } \\
\text { médica melhora } \\
\text { a capacidade } \\
\text { do idoso de } \\
\text { descrever a dor. }\end{array}$ & $\begin{array}{l}\text { Relato de } \\
\text { informações } \\
\text { clínicas relevantes } \\
\text { sobre a dor. }\end{array}$ & $\begin{array}{l}\text { População: Voluntários portadores de } \\
\text { osteoartrite com idade mínima de } 60 \text { anos. } \\
\text { Amostra: } 106 \text { sujeitos (idade média: } 75 \text { anos). } \\
\text { Os sujeitos foram randomizados em } \\
\text { dois grupos para responder ou não a um } \\
\text { questionário com perguntas abertas e fechadas } \\
\text { sobre a queixa de dor, antes da consulta } \\
\text { médica. Durante a consulta médica, ambos os } \\
\text { grupos responderam a um questionário com } \\
\text { perguntas fechadas para avaliação da dor. }\end{array}$ & $\begin{array}{l}\text { Não foram encontradas } \\
\text { diferenças entre os grupos. }\end{array}$ \\
\hline Wetzels et al (18) & $\begin{array}{l}\text { Avaliar a } \\
\text { efetividade de } \\
\text { um programa } \\
\text { educacional } \\
\text { aplicado por } \\
\text { enfermeira } \\
\text { reumatologista, } \\
\text { em uma } \\
\text { única visita } \\
\text { domiciliária. }\end{array}$ & $\begin{array}{l}\text { Estilo de vida, } \\
\text { mobilidade, status } \\
\text { funcional. }\end{array}$ & $\begin{array}{l}\text { População: Sujeitos atendidos por equipe de } \\
\text { saúde domiciliar. } \\
\text { Amostra: } 104 \text { sujeitos ( } 73-75 \text { anos). } \\
\text { Os sujeitos foram randomizados para receber } \\
\text { programa educacional durante uma visita } \\
\text { domiciliária da enfermeira ou panfleto sobre } \\
\text { a doença. }\end{array}$ & $\begin{array}{l}\text { Não foram encontradas } \\
\text { diferenças entre os grupos. }\end{array}$ \\
\hline Taibi et al (19) & $\begin{array}{l}\text { Avaliar a eficácia } \\
\text { e a segurança da } \\
\text { administração } \\
\text { do fitoterápico } \\
\text { extrato de } \\
\text { Valeriana em } \\
\text { sujeitos com } \\
\text { distúrbio do sono. }\end{array}$ & $\begin{array}{l}\text { Horas diárias } \\
\text { de sono, efeitos } \\
\text { adversos. }\end{array}$ & $\begin{array}{l}\text { População: Sujeitos portadores de artrite } \\
\text { atendidos em clínica de reumatologia. } \\
\text { Amostra: } 15 \text { sujeitos ( } 39-53 \text { anos de idade) } \\
\text { Os sujeitos foram randomizados em dois } \\
\text { grupos para receber } 600 \text { mg de extrato de } \\
\text { Valeriana ou placebo, uma hora antes de } \\
\text { dormir. }\end{array}$ & $\begin{array}{l}\text { Não foi encontrada diferença } \\
\text { entre os grupos. }\end{array}$ \\
\hline
\end{tabular}




\begin{tabular}{|c|c|c|c|c|}
\hline Autor & Objetivos & Variáveis & Método & Principais Resultados \\
\hline Baird et al (20) & $\begin{array}{l}\text { Avaliar a } \\
\text { efetividade de } \\
\text { imagem guiada } \\
\text { mais relaxamento. }\end{array}$ & $\begin{array}{l}\text { Dor, mobilidade, } \\
\text { uso de } \\
\text { medicamentos. }\end{array}$ & $\begin{array}{l}\text { População: Voluntários portadores de } \\
\text { osteoartrite, saudáveis à entrada no estudo. } \\
\text { Amostra: } 30 \text { sujeitos (sexo feminino; idade } \\
\text { média: } 70 \text { anos). } \\
\text { Os sujeitos foram randomizados para } \\
\text { praticar duas seções diárias de imagem } \\
\text { guiada e relaxamento ou para receber } \\
\text { orientações sobre atividades diárias e uso de } \\
\text { medicamentos, por } 16 \text { semanas. }\end{array}$ & $\begin{array}{l}\text { O grupo que praticou imagem } \\
\text { guiada mais relaxamento } \\
\text { apresentou redução da dor } \\
\text { (t-test; } p=0,0284) \text { e melhora da } \\
\text { mobilidade }(0,0225) \text { após oito } \\
\text { semanas além de redução no } \\
\text { uso de medicamentos (t-test; } \\
p=0,0189) \text { após } 16 \text { semanas. } O \\
\text { grupo controle não apresentou } \\
\text { mudanças significativas. }\end{array}$ \\
\hline
\end{tabular}

A idade dos sujeitos envolvidos nos estudos esteve na faixa dos 28 aos 80 anos.

Quanto à natureza da intervenção, seis estudos avaliaram a utilização de Terapias Complementares tais como toque terapêutico $(\mathrm{TT})^{(10)}$, terapia espiritual|(11), fitoterapia ${ }^{(19)}$, musicoterapia $^{(13)}$, estimulação elétrica neuromuscular ${ }^{(14)}$, e imagem guia$\mathrm{da}^{(20)}$. Quatro estudos testaram a efetividade do atendimento de enfermeira especializada em reumatologia comparado à equipe multiprofissional à curto $^{(12)}$ e longo prazo $^{(5)}$, sobre $\mathrm{O}$ bem estar dos pacientes ${ }^{(15)}$, e sobre a educação para o controle da doença por meio de visita domiciliar ${ }^{(18)}$. Um estudo investigou a utilidade da utilização de questionário para melhorar a acurácia da coleta de dados acerca dos sintomas apresentados por idosos a fim de favorecer o planejamento e implementação de intervenções específicas ${ }^{(17)}$. Outro estudo ${ }^{(16)}$ comparou a eficácia de dois programas educacionais sendo um direcionado especificamente a portadores de artrite e outro programa direcionado a doentes crônicos de maneira geral.

Em um estudo(10), 25 idosos portadores de artrite residentes em uma instituição asilar foram randomizados em três grupos a fim de se testar a efetividade do toque terapêutico no alívio da dor relacionada à artrite. Os sujeitos do grupo experimental foram submetidos a uma sessão semanal de toque terapêutico por seis semanas. O segundo grupo recebeu mímica (sham) pelo mesmo tempo. O terceiro grupo recebeu cuidados de rotina. Os autores encontraram redução da dor, melhora do status funcional e do bem estar nos sujeitos do grupo experimental $\left(x^{2} ; p=0,001\right)$ comparado aos demais grupos.

Em outro estudo, a utilização de terapia espiritual(11) como coadjuvante do tratamento médico não se mostrou eficaz para modificação da força muscular, rigidez articular, percepção de melhora geral da saúde, dor, e parâmetros hematológicos como contagem de células brancas, hemoglobina, hematócrito, taxa de sedimentação eritrocitária e proteína C-reativa, geralmente alterados devido ao curso da doença. Os autores apontaram dificuldades na composição da amostra. Embora cerca de 500 sujeitos tenham sido considerados elegíveis, a amostra (não calculada) foi estimada em 60, mas somente 29 concluíram o estudo.

A avaliação da influência da música sobre a percepção da dor de idosos com atrite ${ }^{(13)}$ presentou evidências que suportam a sua utilização. Os sujeitos do grupo experimental ouviram uma sequencia de três músicas de Mozart durante 20 minutos por 14 dias: Andantino from Concerto for Flute, Harp, and Orchestra; (2) Overture a le nozze di Figaro e (3) Sonata Symphonie No. 40, primeiro movimento. A primeira e terceira seleções eram de $60 \mathrm{bits} / \mathrm{min}$, a segunda era de 72 bits/min, sendo as músicas de 60 a 80 bits/min consideradas relaxantes. Os sujeitos do grupo controle permaneceram pelo mesmo tempo sentados em silêncio. Os autores concluíram que o grupo experimental referiu menos dor do que o grupo controle nos dias 1,7 e 14, e no seguimento, após a $16^{\mathrm{a}}$ semana, (t-test; $p=0,001)$ avaliado por meio de dois instrumentos: Pain Scale Descriptor e Visual Analogue Scale. Embora nos demais dias de experimento os resultados não tenham sido estatisticamente significativos, os autores consideraram que a intervenção proporcionou alívio progressivo para a queixa álgica.

O estudo do efeito da estimulação elétrica neuromuscular sobre a dor relacionada à osteoartrite do joelho em curto e longo prazo ${ }^{(14)}$, por meio da auto aplicação de uma sessão diária de estimulação (grupo experimental), durante 16 semanas, comparado à participação em um programa educativo em grupo de 12 horas (grupo controle) encontrou resultados favoráveis à estimulação, a curto prazo. Os sujeitos foram submetidos a uma aplicação diária por cerca de 15 minutos, inicialmente com uma intensidade que promovesse a contração de 10 a 20\% da contração isométrica máxima voluntária. Os dados foram coletados nas semanas $0,4,8,12$ e 16, cerca de 15 minutos antes da sessão e 15 minutos após. Os autores encontraram redução significativa da dor referida pelos sujeitos do grupo experimental na medição pós intervenção ( $t$-test; $p<0,001$ ).

Em um estudo ${ }^{(20)}$ realizado para verificar a utilização de imagem guiada com relaxamento para redução da dor, do uso de medicamentos, e melhora da mobilidade de pacientes com osteoartrite foi realizada por meio da randomização de 30 sujeitos em um grupo experimental e outro controle. A intervenção consistia em seguir um roteiro de cinco passos, por meio da escuta de um audiotape, que deveria ser seguido pelos sujeitos por 12 minutos/duas vezes ao dia, durante 4 meses. O grupo controle recebeu um audiotape contendo um roteiro contendo orientações para o uso de medicamentos e para o registro semanal da dor (sham). Os resultados demonstraram que os sujeitos do grupo controle apresentaram redução da dor, durante todo o experimento ( $t$-test; $p=0,0284$ ), melhora da mobilidade, no segundo mês ( $t$-test; $p=0,0225)$, e redução no uso de medicamentos ( $t$-test; $p=0,0008$ ) ao final do experimento. 
Vários estudos se dedicaram a verificar a efetividade do cuidado prestado por enfermeira reumatologista avaliando de diversas variáveis em várias condições.

Um estudo multicêntrico ${ }^{(12)}$ avaliou se o atendimento prestado por enfermeira reumatologista era mais eficaz que o prestado pela equipe da unidade de interação e equipe de atendimento ambulatorial. As variáveis estudadas foram: status funcional e de saúde, qualidade de vida, atividade da doença e satisfação do paciente. Os autores utilizaram várias escalas para mensuração das variáveis: Health Assessment Questionnaire (HAQ), McMaster Toronto Arthritis Patient, Preference Disability Questionnaire, RAND-36 Item Health Survey, Rheumatoid Arthritis Quality of Life Questionnaire, Health Utility Rating Scale, Disease Activity Score. A satisfação dos sujeitos foi mensurada por meio de uma escala visual analógicas (VAS). O estudo foi realizado entre Dezembro de 1996 e Janeiro de 1999 e envolveu 210 pacientes randomizados em três grupos, recrutados nos ambulatórios de reumatologia de seis instituições de saúde. Embora tenha sido realizado por três anos, este estudo apresenta os dados do primeiro ano, apenas. Após a avaliação clínica que ocorreu nas semanas $6,12,26$ e 52, os autores concluíram que houve melhora em três das variáveis (status funcional e de saúde, qualidade de vida, atividade da doença) nos três grupos $\left(x^{2} ; p<0,001\right)$, sem diferença entre os grupos. Somente houve diferença significativa entre os grupos quanto à variável satisfação do paciente, em favor da assistência prestada por enfermeira reumatologista $\left(x^{2} ; p<0,001\right)$.

Ao final do período de três anos, outro estudo foi realizado para verificar os resultados do seguimento ${ }^{(5)}$. Para este estudo, os dados foram colhidos nas semanas 12, 26, 52, 78 e 104. Os mesmos instrumentos e variáveis foram considerados. Os autores concluíram que houve melhora, em todos os grupos, nas seguintes variáveis: qualidade de vida e atividade da doença em todos os grupos ( $t$-test; $p<0,05$ ). $\mathrm{O}$ grupo atendido pela enfermeira especialista apresentou aumento na frequência à procura pelos seus serviços, comparado à procura por serviços de saúde nos demais grupos, na semana 104 (U-test: 0,05).

O impacto da assistência da enfermeira reumatologista sobre o bem estar de sujeitos em monitorização no uso de medicamentos $^{(15)}$ foi avaliado em outro estudo iniciado em 1999 e concluído em 2002. Os 71 sujeitos que participaram do estudo foram randomizados em dois grupos para serem atendidos por enfermeira especialista em reumatologia (grupo experimental) ou por enfermeira não especialista (grupo controle).. Foram utilizados os seguintes instrumentos: Arthritis Impact Measurement Scales e Rheumatology Attitude Index. A coleta de dados ocorreu no $3^{\circ}, 7^{\circ}$ e $12^{\circ}$ mês. Os autores encontraram melhora significativa no grupo experimental no $7^{\circ}$ mês quanto às variáveis impacto da doença $(U$-test; $p<0,03)$ e atividade da doença ( $U$-test; $p<0,0048$ ).

Estilo de vida, mobilidade e status funcional foram as variáveis estudadas em um estudo ${ }^{(18)}$ que avaliou a efetividade de um programa educacional aplicado por enfermeira reumatologista, em uma única visita domiciliária. Cento e quatro sujeitos foram randomizados para receberem um panfleto educativo sobre osteoartrite (grupo controle) ou receberem uma visita para educação relacionada à artrite (grupo experimental). A intervenção consistiu no fornecimento de informações relacionadas ao estilo de vida, estratégias para melhorar a mobilidade e para reduzir a incapacidade funcional. O procedimento era realizado em três etapas: ( ${ }^{\text {a }}$ etapa) entrega, e leitura pelos sujeitos, de um folheto com informações sobre a osteoartrite, gráficos para avaliação do estado de saúde e quatro opções de estilos de vida (exercícios físicos, perda de peso, uso de instrumento para auxilio na deambulação e como utilizar medicamentos para a dor); ( $2^{\mathrm{a}}$ etapa) conversa com a enfermeira sobre a leitura e compreensão da doença, durante 30 minutos; ( $3^{\mathrm{a}}$ etapa) escolha, pelos sujeitos, de uma das quatro opções de estilo de vida contidas no folheto informativo. Três meses após a visita os sujeitos eram entrevistados por telefone para avaliação do resultado da opção escolhida e para receber informações adicionais se necessário. Os autores não encontraram diferença entre os grupos quanto às três variáveis investigadas, a saber: uso de recursos e serviços de saúde, status funcional e mobilidade.

Outro estudo ${ }^{(16)}$ realizado para comparar a eficácia de dois programas educativos sendo um direcionado especificamente a portadores de artrite (Arthritis Self-Help Course - ASHC) e o outro direcionado a doentes crônicos de maneira geral (Chronic Disease Self-Management Program -CDSMP) o fizeram por meio da avaliação das variáveis: uso de serviços de saúde, saúde geral, comportamentos saudáveis, auto cuidado para artrite, incapacidade funcional e dor. Os programas foram aplicados na forma de 48 workshops randomizados para aplicarem a um ou outro dos temas. A avaliação dos resultados foi realizada no início do estudo (baseline) e ao final (pós teste). No total, 416 sujeitos participaram dos programas, sendo que 365 eram afro-americanos e cerca de $75-80 \%$ eram do sexo feminino. Os participantes dos workshops ASHC apresentaram melhora significativa no auto cuidado (t-test; $p=0,004)$, comportamentos saudáveis (prática de exercícios) (t-test; $p=0,004)$, e saúde geral (t-test; $p=0,004)$. Os participantes do workshop CDSMP apresentaram melhora significativa nas variáveis auto cuidado ( $t$-test; $p=0,004$ ), redução da incapacidade funcional (t-test; $p=0,032$ ), saúde em geral (t-test; $\mathrm{p}=0,015)$ e dor (t-test; $\mathrm{p}=0,05)$.

Verificar se responder a um questionário sobre dor especificamente relacionada à artrite (Brief Pain Inventory Short Form -BPI-SF) antes de responder a um questionário contendo questões abertas e fechadas sobre dor em geral auxiliaria idosos portadores de artrite a relatarem suas queixas álgicas, foi objeto de um estudo ${ }^{(17)}$. O grupo experimental respondeu primeiramente o BPI-SF, seguido do segundo questionário; o grupo controle respondeu aos dois questionários na ordem inversa. As respostas ao segundo questionário foram gravadas e analisadas segundo o guideline da Sociedade Americana da Dor para manejo da dor relacionada à artrite. Os autores não encontraram diferença significativa entre os grupos.

\section{DISCUSSÃO}

Esta revisão integrativa da literatura, realizada para responder à questão norteadora "Quais são os cuidados de enfermagem 
que produzem resultados significativos/positivos na saúde das pessoas com artrite?" analisou doze estudos, todos com delineamento experimental, randomização e grupo controle; vários deles não encontraram resultados que sustentassem a utilização das intervenções investigadas.

Entre os estudos que se dedicaram à investigação da eficácia de intervenções para o tratamento da dor relacionada à artrite em idosos, as melhores evidências que encontramos foram: a utilização de musicoterapia para redução da dor de idosos na faixa etária dos 76 anos $^{(13)}$, sendo recomendada uma sessão diária de 20 minutos de audição de músicas que tenham ente 60 a 80 bits/min; e a estimulação neuromuscular elétrica ${ }^{(14)}$, para idosos na faixa etária dos 70 anos, portadores de osteoartrite de joelho, sendo recomendadas sessões diárias de cerca de 15 minutos, inicialmente com uma intensidade que promova contração de 10 a $20 \%$ da contração isométrica máxima voluntária. Os demais estudos ${ }^{(10-11,14,19-20)}$, embora os autores tenham considerado as intervenções eficazes, apresentaram pelo menos um dos problemas metodológicos: amostra insuficiente' controle não efetivo, falta de homogeneidade na amostra e utilização de instrumentos de avaliação inespecíficos; tais dados sugerem a necessidade da realização de novos estudos, com refinamento metodológico, antes que as intervenções posam ser adotadas no cuidado de pacientes com artrite, pelos enfermeiros.

Vários estudos se dedicaram a avaliar o impacto do atendimento de enfermeiro especialista em reumatologia sobre variáveis de saúde relacionadas à artrite ${ }^{(5,12,15,18)}$. Consideramos que os resultados encontrados indicam a importância da atuação deste profissional juntamente à equipe multiprofissional de saúde, no cuidado de pacientes portadores de artrite. Nos casos em que sobrevenha o controle e atividade da doença ${ }^{(15)}$, em especial, há evidências de que o cuidado prestado por este profissional pode trazer resultados adicionais.

Um estudo ${ }^{(18)}$ não encontrou diferença entre a visita domiciliária realizada por enfermeira reumatologista comparada à distribuição de panfleto informativo, para a educação sobre o autocuidado de portadores de artrite suscita reflexões. Não pudemos deixar de comparar esta intervenção com os atendimentos domiciliares existentes no nosso país, onde grande ênfase é dada ao aos resultados obtidos por meio da educação em saúde no domicílio. Uma vez que, no nosso país, o atendimento domiciliar é contínuo e no estudo analisado, houve apenas uma visita ao sujeito no domicílio, podemos hipotetizar que a amplitude do vínculo profissional-cliente estabelecido e o número de visitas podem ter contribuído para este resultado.

Observando os resultados encontrados no estudo que comparou dois programas educativos ${ }^{(16)}$, consideramos que a elaboração de tais programas deve levar em conta os resultados que se pretende atingir. Uma vez que o programa direcionado especificamente para pacientes portadores de artrite mostrou-se mais efetivo para melhorar a prática de exercícios físicos e o programa direcionado para pessoas com problemas crônicos em geral foi considerado mais efetivo para melhorar o controle da dor e a incapacidade funcional, a avaliação do perfil das queixas dos pacientes e de suas necessidades específicas podem fornecer indicações do melhor tipo de programa a se adotar. Considerando a existência do SUS no nosso país, e o crescimento do número de idosos na nossa sociedade, acreditamos que as evidências destes estudos suportam a utilização deste tipo de intervenção na implementação de grupos de idosos em Unidades de Saúde e Estratégias de Saúde da Família, para o cuidado de idosos com artrite.

A utilização de questionário contendo questões fechadas não se mostrou útil para auxiliar os idoso portadores de artrite a relatarem suas queixas álgicas ${ }^{(17)}$, o que revela a necessidade de que os enfermeiros busquem abordagens diferenciadas no atendimento a esses pacientes.

\section{CONSIDERAÇÕES FINAIS}

Com base nos estudos analisados concluímos que há uma grande variedade de intervenções destinadas ao tratamento dos idosos com artrite. Nesta revisão as melhores evidências encontradas para o cuidado direcionado ao alívio deste sintoma foram musicoterapia, estimulação elétrica neuromuscular, toque terapêutico e prática de imagem guiada associada a relaxamento.

Para a utilização da musicoterapia, recomenda-se que seja realizada uma sessão diária durante 20 minutos, utilizando-se músicas que favoreçam o relaxamento, como as músicas clássicas. A utilização de estimulação neuromuscular elétrica mostrou-se efetiva para um tipo específico de artrite: a osteoartrite de joelho. Recomenda-se uma aplicação diária por cerca de 15 minutos, inicialmente com uma intensidade que promova contração de 10 a $20 \%$ da contração isométrica máxima voluntária. A intervenção pode ser realizada pelo próprio paciente, em casa, dede que, antes, tenha sido adequadamente instruído treinado por profissional treinado.

Quanto à implementação de programa educativos, as meIhores evidências sugerem que a utilização de programas educacionais direcionados a idosos na faixa etária dos 65 anos específicos para pacientes portadores de artrite podem ser efetivo para melhorar a prática de exercícios físicos. Programas direcionados aos mesmos sujeitos, mas com conteúdo direcionado a pessoas com problemas crônicos em geral podem ser efetivos para melhorar o controle da dor e a incapacidade funcional.

Embora a literatura estudada não tenha indicado evidências de que a educação em saúde no domicílio seja efetiva para o cuidado de pacientes com artrite, devem ser encorajadas pesquisas futuras, em função da importância dessa atividade no sistema de saúde pública do nosso país.

Consideramos que a presente revisão apresenta como limitação o número de bases de dados selecionadas, e a restrição do idioma, ao inglês, português e espanhol. Entretanto, considerando os critérios de inclusão, as evidências de resultados efetivos de intervenções de enfermagem para alívio/controle de dor trazem contribuição à pratica de enfermagem quanto a identificação e análise das possíveis ações a serem empregadas no planejamento da assistência, nos níveis individual e coletivo, junto à população portadora de artrite. 


\section{REFERÊNCIAS}

1. Fernandes MGM, Solange MCS, Costa FG, Fernandes BM. Qualificadores sócio-demográficos, condições de saúde e utilização de serviços por idosos atendidos na atenção primária. Rev Bras Ciên Saúde 2009;13(2):13-20.

2. Perracini MR. Prevenção e manejo de quedas. In: Ramos LR, coordenação. Guia de geriatria e gerontologia. Barueri: Manole; 2005. p. 193-208.

3. Ferrel, B. Pain management in elderly people. J Am Ger Soc 1991;3:64-73.

4. Fauci A, Braunwald E, Kurt I. Harrison: Med Interna 14. ed. Rio de Janeiro: Mc Graw Hill; 1998. v.2.

5. Tijhuis GJ, Zwinderman AH, Hazes JMW, Breedveld FC, Vlieland TV. Two-year follow-up of a randomized controlled trial of a clinical nurse specialist intervention, inpatient, and day patient team care in rheumatoid arthritis. J Adv Nurs 2003;41(1):34-43.

6. Horta ALM, Ferreira DCO, Zhao LM. Envelhecimento, estratégias de enfrentamento do idoso e repercussões na família. Rev Bras Enferm 2010;63(4):523-8.

7. Domênico EDL, Ide CAC. Enfermagem baseada em evidências: princípios e aplicabilidades. Rev Latino-Am Enferm 2003;11(1):115-8.

8. Mendes KDS, Silveira RCCP, Galvão CM. Revisão integrativa: método de pesquisa para a incorporação de evidências na saúde e na enfermagem. Texto Contexto \& Enferm 2008;17(4):758-64.

9. Clark HD, Wells GA, Huët C, McAlister FA, Salmi LR, Fergusson D, LaupacisA. Assessing the quality of randomized trials: reliability of the Jadad Scale. Controll Clin Trials 1999;20(5):448-52.

10. Gordon A, Merenstein JH, D'amico F, Hudgens D. The effects of therapeutic touch on patients with osteoarthritis of the knee. J Fam Pract 1998;47(4):271-8.

11. Gallez P, Dimmock S, Bird HA. Spiritual healing as adjunct therapy for rheumatoid arthritis. $\mathrm{Br} J$ Nurs 2000;9(11):695-700.

12. Tijhuis Gj, Zwinderman AH, Hazes JM, Van Den Hout WB, Breedveld FC, Vliet Vlieland TP. A randomized comparison of care provided by a clinical nurse specialist, an inpatient team, and a day patient team in rheumatoid arthritis. Arthritis Reum 2002;47(5):525-31.

13. McCaffrey R, Freeman E. Effect of music on chronic osteoarthritis pain in older people. J Adv Nurs 2003;44(5):517-24.

14. Gaines JM, Metter EJ, Talbot LA. The effect of neuromuscular electrical stimulation on arthritis knee pain in older adults with osteoarthritis of the knee. Appl Nurs Res 2004;17(3):201-6.

15. Ryan $S$, Hassel $A B$, Lewis $M$, Farrel $A$. Impact of a rheumatology nurse on the wellbeing of patients attending a drug monitoring clinic. J Adv Nurs 2006;53(3):277-86.

16. Goeppinger J, Armstrong B, Schwartz T, Ensley D, Brady TJ. Self-management education for persons with arthritis: Managing comorbidity and eliminating health disparities. Arthritis Care \& Res 2007;57(6):1081-8.

17. McDonald DD, Shea M, Fedo J, Rose L, Bacon K, Noble K, Stewart J. Older adult pain communication and the Brief Pain Inventory Short Form. Pain Manag Nurs 2008;9(4):154-9.

18. Wetzels R, Van Weel C, Grol R, Wensing M. Family practice nurses supporting self-management in older patients with mild osteoarthritis: a randomized trial. BMC Fam Pract 2008;9(7):1-6.

19. Taibi DM, Bourguignon C, Gill Taylor A. A feasibility study of valrerina extract for sleep disturbance in person with arthritis. Biol Res Nurs 2009;10(4):409-17.

20. Baird CL, Murawski MM, Wu J. Efficacy of guided imagery with relaxation for osteoarthritis symptoms and medication intake. Pain Man Nurs 2010;11(1):56-65. 